\title{
The Effect of Mulberry Fruits Consumption on Lipid Profiles in Hypercholesterolemic Subjects: A Randomized Controlled Trial
}

\author{
Anchalee Sirikanchanarod ${ }^{1}$, Akkarach Bumrungpert ${ }^{1}$, Wiroje Kaewruang ${ }^{2}$, \\ Tipanee Senawong ${ }^{2}$ and Patcharanee Pavadhgul ${ }^{1, *}$
}

\author{
${ }^{1}$ Department of Nutrition, Faculty of Public Health, Mahidol University, 420/1 Rajchavithi Road, Rajchathevee, \\ Bangkok 10400, Thailand \\ ${ }^{2}$ The Queen Sirikit Department of Sericulture, Ministry of Agriculture and Cooperatives, 2175 Phahonyothin \\ Road, Lat Yao, Bangkok 10900, Thailand
}

\begin{abstract}
Mulberry (Morus alba) fruit is abundant nutrients and phytochemicals, especially anthocyanins. Mulberries have anti-oxidant and anti-hyperlipidaemic effects both in vitro and animal models. However, the effect of mulberry fruits on lipid profiles in human is unknown. The aim of this study was to determine the effect of mulberry fruit consumption on lipid profiles in hypercholesterolemic subjects. This study is an experimental study, with a randomized controlled trial. Fifty-eight hypercholesterolemic subjects (aged 30-60 years) were recruited. The intervention group received freeze-

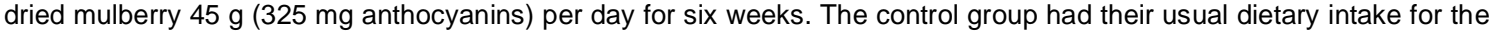
same period of time. After six weeks, mulberry consumption significantly decreased the level of total cholesterol (TC) (- $3.73 \%$ vs. $3.33 \%, p<0.001)$ and low density lipoprotein cholesterol (LDL-C) $(-6.53 \%$ vs. $0.15 \%, p<0.001)$ compared to the control group. No change in triacylglycerol (TAG) and high density lipoprotein cholesterol (HDL-C). Mulberry consumption ameliorates TC and LDL-C concentrations. The mulberry fruits may be an alternative therapy for hypercholesterolemia patients and a cardiovascular disease protective for people in general.
\end{abstract}

Keywords: Mulberry, Morus alba, Anthocyanin, Lipid profiles, Hypercholesterolemic subjects.

\section{INTRODUCTION}

Cardiovascular disease (CVD) is a leading cause of the mortality worldwide. Over $80 \%$ of CVD deaths take place in low- and middle-income countries, which include Thailand [1, 2]. CVD remains one of the most significant diet-related health problems in Thailand and is also the leading cause of death in Thailand. Hyperlipidemia has been identified as a major risk factor for atherosclerosis. Atherosclerosis can lead to other complications which can further cause heart disease or strokes. Several studies have shown that the elevation of low-density lipoprotein cholesterol (LDL-C) levels is related to an increased risk of plaque formation and CVD [3, 4]. Therefore, reducing LDL-C and raising high-density lipoprotein cholesterol (HDL-C) has been considered an important strategy for the prevention and treatment of CVD.

Epidemiological studies suggest that there is an association between diets rich in fruits and vegetables and a reduction in the incidence of CVD [5, 6]. The World Health Organization (WHO) suggests that the consumption of fruits and vegetables in adequate (five

\footnotetext{
*Address correspondence to this author at the Department of Nutrition, Faculty of Public Health, Mahidol University, 420/1 Rajchavithi Road, Rajchathevee, Bangkok 10400, Thailand; Tel: +66 2354 8539; Fax: +66 2640 9839; E-mail: patcharanee@hotmail.com
}

standard portions, $400 \mathrm{~g}$ per day) can prevent the occurrence of chronic diseases such as CVD, hypertension (HT), diabetes mellitus (DM), and cancer. There is a report that low fruit and vegetable consumption contributes to 2.7 million noncommunicable diseases (NCD) related deaths per year [7]. Although fruit and vegetable intake have many health benefits, the consumption of fruit and vegetables in the population remains lower than the recommended levels. More than $80 \%$ of Thai people consume insufficient quantities of fruit and vegetables per day [8]. Dietary intervention is considered a first approach in controlling and treating CVD. Anthocyanins-rich fruits, such as grapes and berries, were reported to have hypocholeserolaemic and antioxidant effects [9]. Berry fruits are the best source of vitamins, minerals, and fiber. Moreover, deep colored fruits and vegetables are not only nutrient-rich but also are good source of phytochemicals, including antioxidants. Streppel et al. [10] found that for every $10 \mathrm{~g}$ per day increment in fiber, there was a $17 \%$ reduction in coronary heart disease mortality and a $9 \%$ reduction in all-cause mortality. In addition, the cohort study reported that anthocyanins intake was associated with a significantly reduced risk of CVD mortality in postmenopausal women [11].

Mulberry belongs to the family of Moraceae and genus Morus. It is a deciduous tree and grows widely

(c) 2016 Lifescience Global 
under various climatic conditions in Asia, Europe, North and South America, and Africa. In Thailand, mulberry is widely grown since it is cultivated easily and provides healthy foliage [12]. It is an economically important plant in the northern and northeast areas of Thailand. In previous times, mulberry production was focused on enhancing the leaves to feed silkworms, the cocoon of which is used in silk production. Recently, Thai people have turned to consuming fruits products of the mulberry. Ripe mulberry fruit is a dark red to purpleblack fruit, which contain antioxidants, being especially high in anthocyanins. The antioxidant activities of anthocyanins are superior to vitamin $\mathrm{C}$ and $\mathrm{E}$. Many studies have found an association between anthocyanin intake, the reduction of damage from free radicals (DPPH, OH, $\mathrm{O}_{2}$, and $\mathrm{H}_{2} \mathrm{O}_{2}$ ), and improved cholesterol concentrations [13-15]. In previous studies, mulberry anthocyanin extracts have demonstrated significant improvements in LDL oxidation, inhibition of lipid biosynthesis and a great capability of scavenging radicals both in vitro and in vivo $[16,17]$.

Since fresh mulberry fruits are soft pulp fruit, they bruise easily and are perishable. Therefore, after harvesting they are highly sensitive to storage conditions and their harvesting seasons are very short. Consequently, we choose freeze-dried mulberries for this study since they contain antioxidant properties, the requisite nutrient composition, an extended shelf life and they also have sensory acceptance by resembling fresh mulberries [18]. However, only a few studies are available about the effects on lipid profiles by mulberry. The majority of the existing literature is limited to in vitro and animal studies. In addition, there are no randomized controlled trials that have reported the effects of mulberry fruit consumption on lipid profiles in humans. Therefore, the aims of this study were to determine freeze-dried mulberries effects on blood lipids in hypercholesterolemic subjects.

\section{METHODS}

\section{Materials}

The mulberry fruits were harvested at the full-ripe stage, and their skin colors were dark purple to black. The ripe mulberry fruits were flash frozen; at the freezing temperatures of $-20^{\circ} \mathrm{C}$ for 48 hours, and then lyophilized using a freeze-drying machine for 24 hours. The freeze-dried mulberries were then placed in sealed food grade packaging.

The quantification of phytochemicals in the freezedried mulberry fruits was verified prior to the commencement of the trial. The total anthocyanins, total polyphenol, and quercitin values, per $45 \mathrm{~g}$, that were found in the freeze-dried mulberries were $326.25 \pm 1.43,482.40 \pm 1.68,0.62 \pm 1.95 \mathrm{mg}$, respectively.

\section{Subjects}

Sixty hypercholesterolemic subjects aged 30-60 years were recruited by an advertisement in the research area. The subjects were considered to have a fasting total cholesterol level of $\geq 200 \mathrm{mg} / \mathrm{dL}$ or LDL cholesterol $\geq 130 \mathrm{mg} / \mathrm{dL}$. They were excluded if they had any chronic diseases, including cancer, CVD, diabetes mellitus, hypertension, liver disease, renal disease or thyroid disorders. Also, subjects who were consuming steroidal medications, antioxidants, and lipid-lowering dietary supplements or alcohol or were currently smokers, pregnant or lactating were excluded. The study was approved by the Ethical Review Committee for Human Research, in the Faculty of Public Health, Mahidol University No.MUPH 2014-094. All subjects provided written informed consent.

\section{Study Design}

This study was a randomized controlled trial. After the screening for the exclusion and inclusion criteria, the eligible subjects were randomized by stratified random sampling to the mulberry group $(n=30)$ or the control group $(n=30)$. The mulberry group consumed $45 \mathrm{~g}$ of freeze-dried mulberry (equivalent $160 \mathrm{~g}$ fresh mulberries, $325 \mathrm{mg}$ anthocyanins) per day for six weeks. Our mulberry dose was selected based on previously reported anthocyanins interventions in a human study, where a daily intake of $320 \mathrm{mg}$ anthocyanins was shown to safe and effective in improving the level of lipid profiles [19]. For seven days preceeding and during the trial, all subjects were instructed to avoid consuming anthocyanin-rich foods and beverages (e.g. berries, red grapes, berry juice); a list of these food and beverages was given to each subjects. The subjects maintained their usual diet, physical activity, and lifestyle patterns during the period of study. In addition, they were also asked to record their daily food intake. Subjects returned for follow-up visits at weeks 2, 4 and 6 of the trial. Their anthropometric measurements, including body weight, height, waist circumference and blood pressure were performed by trained personal and blood draws were performed by a professional nurse at the baseline and the end of study. No member of laboratory staff knew the treatment group. 


\section{Anthropometric Assessment}

Body weight, body fat and visceral fat were determined using a Body fat analyzer scale Model HBF-356 (Omron) that measures using the Bioelectrical Impedance (BI) method.

\section{Biochemical Analysis}

Blood samples were collected at both the baseline and at the end of intervention in all subjects at the same time. The subjects were required to fast overnight for at least 12 hours to enable blood sample collection in the morning. A blood sample of $5 \mathrm{ml}$ were collected for determining the lipid profiles including total cholesterol (TC), triacylglycerol (TAG), high density lipoprotein cholesterol (HDL-C), and low density lipoprotein cholesterol (LDL-C). Serum total cholesterol and TAG were measured using an enzymatic colorimetric test. HDL-C and LDL-C were measured using a homogeneous enzymatic colorimetric.

\section{Dietary Analyses}

This study used the three-day food records to assess the daily food intake (including 2 workdays and 1 weekend) at the baseline, at week 3 and again at week 6 of the study. The subjects also received training on accurate food record completion; the details included recording type and amount of food consumed and the cooking method. The subjects estimated their food portion sizes by using food models or common household utensils such as cups, table-spoons, or a tea-spoon and cup. The subjects recorded all foods and beverages consumed each day starting from when they wake up and tried to list the foods/ beverages immediately after eating or drinking. Portion size measures were converted into grams. Food intake records were analyzed by using an INMUCAL-Nutrients computer program.

\section{Statistical Analysis}

The difference on lipid profiles and anthropometry between the mulberry group and the control group were compared by using an Independent samples $t$ test and calculating the differences in lipid profiles and anthropometry in the mulberry group (pre-post test) by using a paired $t$-test. The analysis of the dietary intakes for each group was by One-way ANOVA. Data were analyzed using SPSS 18.0 for Windows statistical analysis software and statistical significance was accepted at $p$-value $<0.05$

\section{RESULTS}

\section{Anthropometric Characteristics and Dietary Intake}

Sixty hypercholesterolemic subjects were included in the study. Two subjects in the control group lost follow-up during the study. The characteristics of the fifty-eight subjects who completed the study are shown Table 1. The gender ratio of subjects (male: female) were 4: 26 and 4: 24 in the mulberry and the control

Table 1: Changes in the Anthropometrics at Baseline (Week 0) and the End of Study (Week 6) in the Mulberry and Control Groups ${ }^{1}$

\begin{tabular}{|c|c|c|c|c|c|}
\hline Variables & Group & Week 0 & $p-$ value $^{2}$ & Week 6 & $p-$ value $^{3}$ \\
\hline \multirow[t]{2}{*}{ Body weight $(\mathrm{kg})$} & Mulberry & $58.67 \pm 11.19$ & \multirow[t]{2}{*}{0.301} & $58.60 \pm 11.05$ & \multirow[t]{2}{*}{0.246} \\
\hline & Control & $62.38 \pm 15.67$ & & $62.72 \pm 15.48$ & \\
\hline \multirow[t]{2}{*}{$\mathrm{BMI}\left(\mathrm{kg} / \mathrm{m}^{2}\right)$} & Mulberry & $23.87 \pm 3.87$ & \multirow[t]{2}{*}{0.454} & $24.02 \pm 3.86$ & \multirow[t]{2}{*}{0.464} \\
\hline & Control & $24.80 \pm 5.41$ & & $24.92 \pm 5.32$ & \\
\hline \multirow[t]{2}{*}{$\%$ body fat } & Mulberry & $32.44 \pm 5.28$ & \multirow[t]{2}{*}{0.471} & $32.34 \pm 5.44$ & \multirow[t]{2}{*}{0.563} \\
\hline & Control & $31.34 \pm 6.14$ & & $31.09 \pm 6.81$ & \\
\hline \multirow[t]{2}{*}{$\%$ visceral fat } & Mulberry & $7.17 \pm 4.04$ & \multirow[t]{2}{*}{0.621} & $6.93 \pm 3.92$ & \multirow[t]{2}{*}{0.433} \\
\hline & Control & $7.99 \pm 5.93$ & & $8.00 \pm 5.55$ & \\
\hline \multirow{2}{*}{$\begin{array}{c}\text { Waist } \\
\text { circumference }(\mathrm{cm})\end{array}$} & Mulberry & $81.67 \pm 9.87$ & \multirow[t]{2}{*}{0.667} & $83.22 \pm 9.55$ & \multirow[t]{2}{*}{0.534} \\
\hline & Control & $82.98 \pm 12.90$ & & $85.00 \pm 12.06$ & \\
\hline \multirow[t]{2}{*}{$\mathrm{SBP}(\mathrm{mmHg})$} & Mulberry & $118.17 \pm 15.18$ & \multirow[t]{2}{*}{0.844} & $115.77 \pm 13.40$ & \multirow[t]{2}{*}{0.745} \\
\hline & Control & $119.00 \pm 16.82$ & & $116.96 \pm 14.55$ & \\
\hline \multirow[t]{2}{*}{$\mathrm{DBP}(\mathrm{mmHg})$} & Mulberry & $77.37 \pm 9.31$ & \multirow[t]{2}{*}{0.868} & $74.23 \pm 10.44$ & \multirow[t]{2}{*}{0.869} \\
\hline & Control & $76.93 \pm 10.62$ & & $73.79 \pm 10.03$ & \\
\hline
\end{tabular}

${ }^{1}$ Data are shown as mean \pm SD.

${ }^{2}$ There were no significant differences between the two groups at baseline for any variable by the independent-sample $t$ test.

${ }^{3}$ There were no significant differences between the two groups at the end of study for any variable by the independent-sample $t$ test. 
Table 2: Dietary Nutrient Intakes of the Subjects at Baseline, Week 3, and Week 6 of Study ${ }^{1}$

\begin{tabular}{|c|c|c|c|c|}
\hline & & Mulberry & Control & $p-$ value $^{3}$ \\
\hline \multirow[t]{2}{*}{ Energy (kcal/d) } & Week 0 & $1,265.0 \pm 279.1$ & $1,243.9 \pm 288.6$ & 0.783 \\
\hline & Week 6 & $1,163.7 \pm 295.7$ & $1,183.3 \pm 230.9$ & 0.785 \\
\hline$p-$ value $^{2}$ & & 0.393 & 0.702 & \\
\hline \multirow{2}{*}{$(g / d)$} & Week 3 & $175.8 \pm 47.0$ & $181.9 \pm 53.0$ & \\
\hline & Week 6 & $170.1 \pm 41.8$ & $171.2 \pm 44.2$ & 0.925 \\
\hline$p-$ value $^{2}$ & & 0.305 & 0.422 & \\
\hline (\% of energy) & Week 0 & $59.2 \pm 8.2$ & $58.9 \pm 7.4$ & 0.872 \\
\hline \multicolumn{5}{|l|}{ Protein } \\
\hline \multirow[t]{3}{*}{$(g / d)$} & Week 0 & $43.1 \pm 14.2$ & $43.1 \pm 12.2$ & 0.984 \\
\hline & Week 3 & $41.5 \pm 12.7$ & $41.9 \pm 10.5$ & \\
\hline & Week 6 & $44.1 \pm 17.5$ & $41.7 \pm 11.8$ & 0.560 \\
\hline$p-$ value $^{2}$ & & 0.793 & 0.890 & \\
\hline \multirow[t]{3}{*}{ (\% of energy) } & Week 0 & $13.7 \pm 2.6$ & $14.0 \pm 3.1$ & 0.725 \\
\hline & Week 3 & $14.1 \pm 2.3$ & $14.0 \pm 2.9$ & \\
\hline & Week 6 & $14.7 \pm 2.9$ & $14.8 \pm 4.1$ & 0.977 \\
\hline \multirow[t]{3}{*}{ (\% of energy) } & Week 0 & $26.6 \pm 6.6$ & $25.5 \pm 6.0$ & 0.543 \\
\hline & Week 3 & $28.0 \pm 9.0$ & $27.2 \pm 6.3$ & \\
\hline & Week 6 & $26.2 \pm 6.8$ & $28.1 \pm 6.1$ & 0.286 \\
\hline$p-$ value $^{2}$ & & 0.614 & 0.317 & \\
\hline \multicolumn{5}{|l|}{ Cholesterol (mg/d) } \\
\hline & Week 0 & $202.4 \pm 100.2$ & $203.1 \pm 94.3$ & 0.979 \\
\hline & Week 3 & $199.4 \pm 118.8$ & $201.6 \pm 87.7$ & \\
\hline & Week 6 & $195.0 \pm 118.8$ & $195.9 \pm 92.8$ & 0.973 \\
\hline$p-$ value $^{2}$ & & 0.968 & 0.957 & \\
\hline \multicolumn{5}{|l|}{ Fiber (g/d) } \\
\hline & Week 0 & $10.7 \pm 6.9$ & $8.0 \pm 4.9$ & 0.096 \\
\hline & Week 3 & $19.4 \pm 5.9$ & $8.3 \pm 3.7$ & \\
\hline & Week 6 & $18.4 \pm 4.3$ & $7.7 \pm 3.8$ & $<0.001^{*}$ \\
\hline$p-$ value $^{2}$ & & $<0.001^{*}$ & 0.865 & \\
\hline
\end{tabular}

${ }^{1}$ Data are shown as mean \pm SD.

${ }^{2}$ Analysis of the difference dietary intakes for each group at the baseline, week 3 , and week 6 of study for any variables by One-way ANOVA.

${ }^{3}$ Analysis of the difference in the dietary intakes for between the two groups at the baseline and the end of study for any variable by independent-sample $t$ test.

*Significant differences, $p<0.001$. 
groups, respectively. The average age of the subjects in mulberry group and control group were $46.57 \pm 8.46$ and $44.96 \pm 8.70$ years, respectively. At the baseline, no differences were observed in age and anthropometric characteristics between the mulberry and the control group. Similarly, there were also no differences in body weight, body mass index, body fat percentage, visceral fat, waist circumference and blood pressure after the end of study (Table 1). The mulberry group had a significantly higher dietary fiber intake than at the baseline point and the control group at the end of week 6 . However there were no differences in energy, carbohydrate, protein, fat and cholesterol intake between both groups and along the period of time (Table 2). During the first week of the study, seven subjects had mild gastrointestinal side effects including flatulence and discomfort in the abdomen but did not require any treatment and was possibly as a result of additional fiber in the freeze-dried mulberry intake.

\section{Effects of Freeze-Dried Mulberries Consumption on Fasting Serum Lipid Profiles}

As shown in Table 3, the baseline concentrations of levels TC, LDL-C, HDL-C and TAG did not differ between the two groups. In the mulberry group, there were a significantly decreased serum TC from $235.07 \pm$ 28.10 to $225.83 \pm 24.26(p<0.001)$ and serum LDL-C from $167.47 \pm 29.32$ to $156.07 \pm 24.70(p<0.001)$ after the six weeks freeze-dried mulberry intake. There were no differences in HDL-C and TAG levels. The control

Table 3: Changes in Lipid Profiles at Baseline (Week 0) and the End of Study (Week 6) in the Mulberry Group and the Control Group ${ }^{1}$

\begin{tabular}{|c|c|c|c|c|}
\hline \multirow{2}{*}{ Lipid profiles $(\mathbf{m g} / \mathbf{d L})$} & Group & Week 0 & Week 6 & Mean change \\
\hline \hline \multirow{2}{*}{ TC $^{*}$} & Mulberry & $235.07 \pm 28.10$ & $225.83 \pm 24.26$ & $-9.23 \pm 10.75$ \\
\cline { 2 - 5 } & Control & $237.26 \pm 32.72$ & $244.70 \pm 33.12$ & $7.44 \pm 12.13$ \\
\hline \multirow{2}{*}{ LDL-C* } & Mulberry & $167.47 \pm 29.32$ & $156.07 \pm 24.70$ & $-11.40 \pm 12.04$ \\
\cline { 2 - 5 } & Control & $166.59 \pm 34.02$ & $166.48 \pm 32.64$ & $-0.11 \pm 8.77$ \\
\hline \multirow{2}{*}{ HDL-C } & Mulberry & $61.13 \pm 12.59$ & $62.57 \pm 13.66$ & $1.43 \pm 4.96$ \\
\cline { 2 - 6 } & Control & $65.39 \pm 15.86$ & $66.14 \pm 15.96$ & $0.75 \pm 7.33$ \\
\hline \multirow{2}{*}{ TAG } & Mulberry & $117.03 \pm 41.21$ & $109.28 \pm 43.65$ & $-7.76 \pm 30.69$ \\
\cline { 2 - 5 } & Control & $100.50 \pm 31.97$ & $97.31 \pm 30.74$ & $-3.19 \pm 25.40$ \\
\hline
\end{tabular}

Abbreviations. TC: total cholesterol, LDL-C: low density lipoprotein cholesterol, HDL-C: high density lipoprotein cholesterol, TAG: triacylglycerol ${ }^{1}$ Data are shown as mean \pm SD

${ }^{*}$ Significant differences, $p<0.001$ assessed by independent-sample $t$ test.

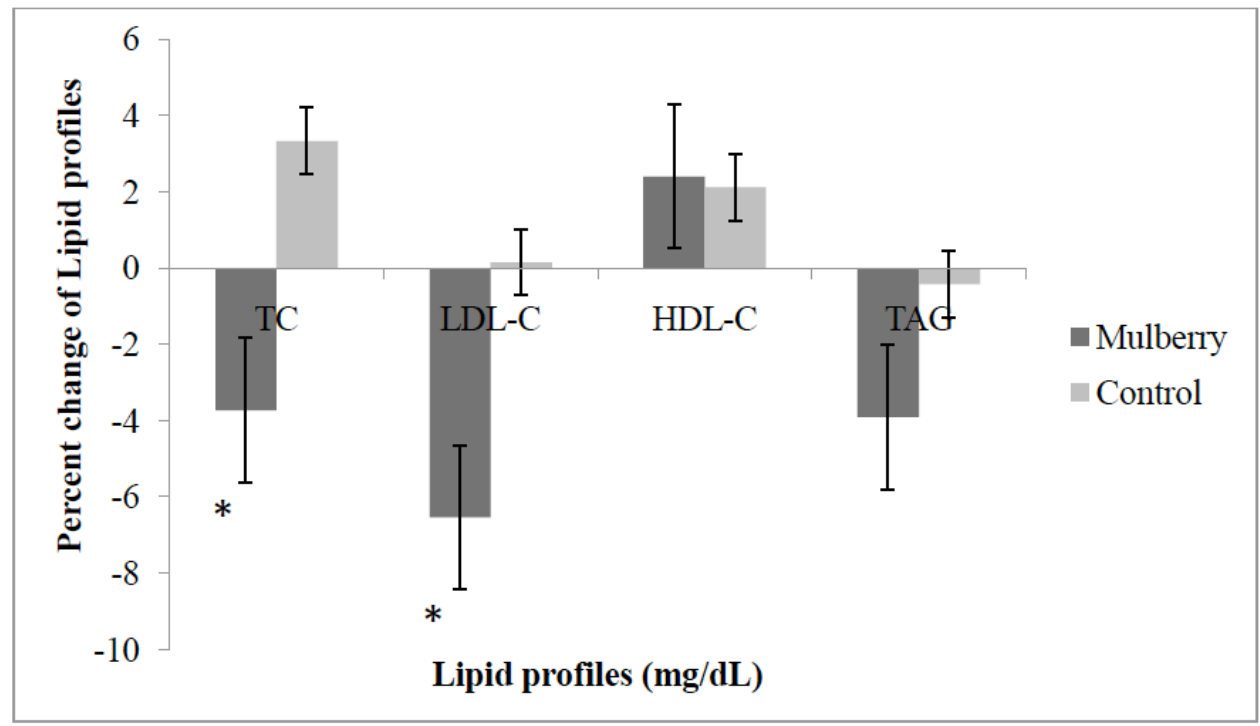

Figure 1: Percentage change in lipid profiles between the mulberry group and the control group.

${ }^{*}$ Significant differences, $p<0.001$. 
group significantly increased serum TC from $237.26 \pm$ 32.72 to $244.70 \pm 33.12(p=0.004)$ and there was no change in serum TC, LDL-C and HDL-C. We observed significant differences in the changes of LDL-C by $6.53 \%$ and TC by $3.73 \%(p<0.001)$ levels between the mulberry and control group, while TAG and HDL-C did not change (Figure 1).

\section{DISCUSSION}

In the present study, we investigated the effect of mulberry fruits consumption on blood lipid profiles in hypercholesterolemic subjects. Our data showed that six weeks' of freeze-dried mulberry $(325 \mathrm{mg}$ anthocyanins) intake resulted in significant reducing serum TC and LDL-C by $3.73 \%, 6.53 \%$, respectively. It had no effect on TAG and HDL-C. Likewise, two previous studies have demonstrated that purified anthocyanins derived from bilberry and black currant supplementation decreased LDL-C (13.6\%), increased HDL-C (13.7\%) for eight weeks in dyslipidemic subjects [19] and lowered the LDL-C (10.4\%), and raised the HDL-C (14\%) in six months in adults with hypercholesterolemia. There were no significant differences in the levels of TAG and TC [20]. Similarly, the results have consistently been observed with freeze-dried strawberry powder (320 mg anthocyanins) in subjects with metabolic syndrome as well [21, 22]. On the other hand, Curtis et al. [23] was conducted to investigate the effects of elderberry $(500 \mathrm{mg}$ anthocyanins) on the biomarkers of CVD risk and liver and kidney functions for 12 weeks in healthy postmenopausal women. There was no significant change in the biomarker of inflammatory (CRP, TNF $\alpha$ ) and blood lipid profiles. The mulberry fruits are good source of natural bioactive compounds or phytochemicals that exhibit pharmacological and biological effects on human health. Mulberries have plenty of anthocyanins (210-294 mg per $100 \mathrm{~g}$ fresh weight) [24], which is equal to that of bilberries, blueberries and black currants. Mulberries contained higher amounts of anthocyanins than cranberries and strawberries [25]. The major anthocyanins found in mulberries are cyanidin-3-glucoside (cy-3-glc) and cyanidin-3-rutinoside (cy-3-rut) [26, 27], while bilberries, blueberries, black currant, strawberries, elderberries are delphinidin (dp), malvidin (mv), delphinidin (dp), pelargonindin (pl), cyaniding (cy), respectively [28]. It might be possible that the cholesterol-lowering effects of berry fruits depend on the chemical structure of the anthocyanins, the duration of the invention, and the characteristics of subjects.
Anthocyanins from mulberry have been shown to affect lipid metabolism in cellular culture and animal models of dyslipidemia. The results from the study by Yang et al. [29] determined the hypolipidemic and antioxidant effects of freeze-dried powder mulberry (MFP) fruit in hyperlipidaemia rats. After the end of trial, rats fed MFP on high-fat diet significantly reduced the levels of serum and liver TC, TAG, LDL-C, while HDL-C and anti-oxidant enzyme activity (SOD, GSH-Px) were significantly increased. Moreover, the mulberry water extracts (MWEs) and mulberry anthocyanins-rich extracts (MACs) have a great ability to scavenge free radicals, inhibit LDL oxidation, decrease macrophage deaths and foam cell formation, which was induced by oxidized LDL-C, while the efficacy of the MACs was 10 times greater than that of MWEs [16].

Interestingly, several studied have been found the mechanisms of the cholesterol-lowering effects of mulberry were as follows: (i) they blocked the uptake of cholesterol into Caco-2 cells (about 25\% inhibition) [30], (ii) inhibited cholesterol ester transfer protein (CETP) activity [19], (iii) inhibited cellular lipid accumulation through activating the AMPK pathway, (iv) suppressed the lipogenic enzyme , (v) enhanced the gene expression of the LDL receptor and (vi) stimulated fatty acid oxidation [17, 20, 31].

Nevertheless, the mulberry fruits are not only a good source of phytochemicals but also plenty of vitamins, minerals, and dietary fiber. The predominant mineral elements of these fruits are potassium, followed by the calcium, sodium, magnesium, phosphorus, iron, zinc, copper, and manganese [32]. Koca et al. [26] reported that calcium, sodium, and iron contents of mulberries are higher than values in other berries, including strawberry, redcurrant, blackcurrant, raspberry, blueberry and blackberry. Dietary calcium is one mineral which is able to up-regulate the gene of cholesterol-7a-hydroxylase (CYP7A1), a rate-limiting enzyme in the synthesis of bile acids in the hepatic system that brings about raised bile production and the excretion of fecal cholesterol. It also decreases plasma CETP activity, which leads to a reduction in the liver and plasma cholesterol levels [33].

Moreover, the freeze-dried mulberry provides approximately $24.3 \mathrm{~g}$ fiber $/ 100 \mathrm{~g}$ freeze-dried mulberry [34]. Hypocholesterolaemic action was, at least partly, found in our study may have been due to the increase in dietary fiber intake from the baseline of about $10 \mathrm{~g}$ per day during the six weeks of the study. The results of a cohort study by Castro et al. [35] following 316 
Japanese-Brazilians subjects over seven years observed a decrease of $12.5 \mathrm{mg} / \mathrm{dL}$ in the serum TC levels for each increase of $10 \mathrm{~g}$ in the consumption of dietary fiber. In addition, the American Dietetic Association recommends a total dietary fiber intake of $14 \mathrm{~g}$ per $1000 \mathrm{kcal}$ from foods in order to reduce blood lipid profiles and other risk factors of CVD [36]. Hence, the synergistic effects of anthocyanins and fiber in mulberry fruits are possibly the reasons for decreasing serum TC, LDL-C levels in the hypercholesterolemic subjects. Mulberries are remarkable fruits because they provide abundant nutrients, at a low cost, but are still rich in anthocyanins with an equivalence to other berries. It is estimated that $100 \mathrm{~g}$ of mulberries provide anthocyanins equal to $500 \mathrm{~g}$ of strawberries. The advantages of freeze-dried mulberries consumption are eat as a snack, delicious, healthy and an excellent choice for long-term food storage, which do not waiting for that fruit-producing season. Our study is these first to our knowledge indicate that the potential of mulberry fruits to ameliorate on blood lipid profiles in human intervention. Since most current cholesterol-lowering drugs are expensive and have potential side effects, mulberry fruits could be an alternative therapy for hypercholesterolemia patients and a CVD protective for people in general. Certain limitations of this study include subjects consisting primarily of women and it examined mulberries intake over a short period of time. Further prospective studies should be undertaken to evaluate the impact of fresh mulberry consumption on lipid profiles and different cardiovascular benefits. These studies may also be used to confirm the benefits in men.

In conclusion, we have provided evidence that the consumption of mulberry fruits significantly lowered TC and LDL-C concentrations in hypercholesterolemic subjects. The current study indicates that mulberry fruit is natural source of antioxidants and a great way to boost the fiber content in dietary intake, which improve blood lipids and benefits cardiovascular health in humans.

\section{ACKNOWLEDGEMENTS}

The authors are thankful the Queen Sirikit Department of Sericulture, Ministry of Agriculture and Cooperatives for their financial support of this research and Chul Thai Silk Co., Ltd. for freeze-dried mulberry in this study.

\section{REFERNCES}

[1] World Health Organization. Global status report on noncommunicable disaeses 2014. Geneva: WHO Press;
2014.

[2] Mathers CD, Loncar D. Projections of global mortality and burden of disease from 2002 to 2030. PLoS Med 2006; 3: 2011-30.

[3] Hawkins MA. Markers of increased cardiovascular risk: are we measuring the most appropriate parameters? Obes Res 2004; 12(Suppl 2): S107-S14.

[4] Duarte MM, Rocha JB, Moresco RN, Duarte T, Da Cruz IB, Loro VL, et al. Association between ischemia-modified albumin, lipids and inflammation biomarkers in patients with hypercholesterolemia. Clin Biochem 2009; 42: 666-71. http://dx.doi.org/10.1016/j.clinbiochem.2009.01.010

[5] Duachet L, Amouyel P, Dallongeville J. Fruits, vegetables and coronary heart disease. Nat Rev Cardiol 2009; 6: 599-608. http://dx.doi.org/10.1038/nrcardio.2009.131

[6] Gan $\mathrm{Y}$, Tong $\mathrm{X}$, Li L, Cao S, Yin X, Gao C, et al. Consumption of fruit and vegetable and risk of coronary heart disease: A meta-analysis of prospective cohort studies. Int J Cardiol 2015; 183: 129-37. http://dx.doi.org/10.1016/j.ijcard.2015.01.077

[7] Hall JN, Moore S, Harper SB, Lynch JW. Global variability in fruit and vegetable consumption. Am J Prev Med 2009; 36(5): 402-9.

http://dx.doi.org/10.1016/j.amepre.2009.01.029

[8] Akepalakorn W, editor. The Thailand National Health Examination Survey IV 2008-2009. Nonthaburi: The Graphico System, 2011.

[9] Zafra-Stone S, Yasmin T, Bagchi M, Chatterjee A, Vinson JA, Bagchi D. Berry anthocyanins as novel antioxidants in human health and disease prevention. Mol Nutr Food Res 2007; 51: 675-83. http://dx.doi.org/10.1002/mnfr.200700002

[10] Streppel MT, Ocké MC, Boshuizen HC, Kok FJ, Kromhout D. Dietary fiber intake in relation to coronary heart disease and all-cause mortality over $40 \mathrm{y}$ : the Zutphen Study. Am J Clin Nutr 2008; 88(4): 1119-25.

[11] Mink PJ, Scrafford CG, Barraj LM, Harnack L, Hong CP, Nettleton JA, et al. Flavonoid intake and cardiovascular disease mortality: a prospective study in postmenopausal women. Am J Clin Nutr 2007; 85: 895-909.

[12] Aramwit $P$, Bang N, Srichana T. The properties and stability of anthocyanins in mulberry fruits. Food Res Int 2010; 43: 1093-7.

http://dx.doi.org/10.1016/j.foodres.2010.01.022

[13] Jeszka JW. Food colorants.In: Sikorski ZE,editor. Chemical and functional Properties of food Components. $3^{\text {rd }}$ ed. New York: Taylor \& Francis Group; 2007; p. 245-74.

[14] Castañeda-Ovando $A$, de Lourdes Pacheco-Hernández $M$, Páez-Hernández ME, Rodríguez JA, Galán-Vidal CA. Chemical studies of anthocyanins: A review. Food Chem 2009; 113: 859-71. http://dx.doi.org/10.1016/j.foodchem.2008.09.001

[15] Zhao CL, Guo1 HC, Dong ZY, Zhao Q. Pharmacological and nutritional activities of potato anthocyanins. Afr $\mathrm{J}$ Pharm Pharmacol 2009; 2: 463-8.

[16] Liu LK, Lee HJ, Shih YW, Chyau CC, Wang CJ. Mulberry anthocyanin extracts inhibit LDL oxidation and macrophagederived foam cell formation induced by oxidative LDL. J Food Sci 2008; 73(6): 113-21.

http://dx.doi.org/10.1111/j.1750-3841.2008.00801.x

[17] Ou TT, Hsu MJ, Chan KC, Huang CN, Ho HH, Wang CJ. Mulberry extract inhibits oleic acid-induced lipid accumulation via reduction of lipogenesis and promotion of hepatic lipid clearance. J Sci Food Agric 2011; 91: 2740-8.

http://dx.doi.org/10.1002/jsfa.4489

[18] Pansuwan S, Nuipirom W, Apinyanuwat S, Numphanon $\mathrm{H}$, Jomduang S. Study on product of mulberry freeze drying. Annual research report of sericulture 2007, Bangkok; 2008: pp. 285-93. 
[19] Qin Y, Xia M, Ma J, Hao Y, Liu J, Mou H, et al. Anthocyanin supplementation improves serum LDL- and HDL- cholesterol concentrations associated with the inhibition of cholesteryl ester transfer protein in dyslipidemic subjects. Am J Clin Nutr 2009; 90: 485-92. http://dx.doi.org/10.3945/ajcn.2009.27814

[20] Zhu Y, Ling W, Guo H, Song F, Ye Q, Zou T, et al. Antiinflammatory effect of purified dietary anthocyanin in adults with hypercholesterolemia: A randomized controlled trial. Nutr Metab Cardiovasc Dis 2013; 23: 843-49. http://dx.doi.org/10.1016/j.numecd.2012.06.005

[21] Basu A,Wilkinson M, Penugonda K, Simmons B, Betts NM, Lyons TJ. Freeze-dried strawberry powder improves lipid profile and lipid peroxidation in women with metabolic syndrome: baseline and post intervention effects. Nutr $\mathrm{J}$ 2009; 8: 1-7. http://dx.doi.org/10.1186/1475-2891-8-43

[22] Basu A, Fu DX, Wilkinson M, Simmons B, Wu M, Betts NM, et al. Strawberries decrease atherosclerotic markers in subjects with metabolic syndrome. Nutr Res 2010; 30: 462-9. http://dx.doi.org/10.1016/j.nutres.2010.06.016

[23] Curtis PJ, Kroon PA, Hollands WJ, Walls R, Jenkins G, Kay $C D$, et al. Cardiovascular disease risk biomarkers and liver and kidney function are not altered in postmenopasausal women after ingesting an elderberry extract rich in anthocyanins for 12 weeks. J Nutr 2009; 139(12): 2266-71. http://dx.doi.org/10.3945/jn.109.113126

[24] Numphanon H, Nuipirom W, Apinyanuwat $\mathrm{S}$, et al. Production development of retort pouch mulberry in syrup. Annual research report of sericulture 2007, Bangkok; 2008: pp. 572-604.

[25] de Pascual-Teresa S, Moreno DA, Garcia-Viguera C. Flavanols and anthocyanins in cardiovascular Health: A review of current evidence. Int J Mol Sci 2010; 11: 1679-703. http://dx.doi.org/10.3390/ijms11041679

[26] Koca I, Tural S. Physico-chemical and antioxidant properties of cornelian cherry fruits (Cornus mas L.) grown in Turkey. Sci Hort 2008; 116: 362-6.

[27] Hassimotto NM, Genovese MI, Lajolo FM. Absorption and metabolism of cyanidin-3-glucoside and cyanidin-3-rutinoside extracted from wild mulberry (Morus nigra L.) in rats. Nutr Res 2008; 28: 198-207.

http://dx.doi.org/10.1016/j.nutres.2007.12.012
[28] Valenti L, Riso P, Mazzocchi A, Porrini M, Fargion S, Agostoni C. Dietary anthocyanins as nutritional therapy for nonalcoholic fatty liver disease. Oxid Med Cell Longev 2013; $1-8$.

http://dx.doi.org/10.1155/2013/145421

[29] Yang X, Yang L, Zheng H. Hypolipidemic and antioxidant effects of mulberry (Morus alba L.) fruit in hyperlipidaemia rats. Food Chem Toxicol 2010; 48: 2374-9. http://dx.doi.org/10.1016/j.fct.2010.05.074

[30] Duangjai A, Ingkaninan K, Limpeanchob. Potential mechanisms of hypocholesterolemic effect of Thai spices/dietary extracts. Nat Prod Res 2011; 25(4): 341-352. http://dx.doi.org/10.1080/14786411003754249

[31] Liu LK, Chou FP, Chen YC Chyau CC, Ho HH, Wang CJ. Effects of mulberry (Morus alba L.) extracts on lipid homeostasis in vitro and in vivo. J Agric Food Chem 2009; 26(57): 7605-11. http://dx.doi.org/10.1021/jf9014697

[32] Liang L, Wu X, Zhu M, Zhao W, Li F, Zou Y, et al. Chemical composition, nutritional value, and antioxidant activities of eight mulberry cultivars from China. Pharmacogn Mag 2012; 8(31): 215-24. http://dx.doi.org/10.4103/0973-1296.99287

[33] Ma KY, Yang N, Jiao R, Peng C, Guan L, Huang Y, et al. Dietary calcium decreases plasma cholesterol by downregulation of intestinal Niemann-Pick $\mathrm{C} 1$ like 1 and microsomal triacylglycerol transport protein and up-regulation of CYP7A1 and ABCG 5/8 in hamsters. Mol Nutr Food Res 2011; 55: 247-58. http://dx.doi.org/10.1002/mnfr.201000161

[34] Ozguven MG, Ozcelik B. Dried mulberries:phytochemicals and health effects. In: Alasalvar C, Shahidi F, editors. Dried fruits phytochemicals and health effects. Oxford, John Wiley \& Sons; 2013; pp. 145-60.

[35] de Castro TG, Gimeno SG, Ferreira SR, Cardoso MA; Japanese-Brazilian Diabetes Study Group. Association of dietary fiber with temporal changes in serum cholesterol in Japanese-Brazilians. J Nutr Sci Vitaminol 2006; 52: 205-10.

[36] Marlett JA, McBurney MI, Slavin JL; American Dietetic Association. Position of the American Dietetic Association: health implications of dietary fiber. J Am Diet Assoc 2002; 102: 993-1000.

http://dx.doi.org/10.1016/S0002-8223(02)90228-2 